\title{
Modelling Stellar Rotation for Optical Long Baseline Interferometry
}

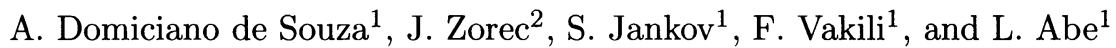 \\ ${ }^{1}$ Département d'Astrophysique de l'Université de Nice/Sophia-Antipolis, \\ CNRS UMR 6525, France \\ 2 Institut d'Astrophysique de Paris, CNRS, 98bis Boulevard Arago, \\ F-75014 Paris, France
}

\begin{abstract}
.
Optical long baseline interferometry is a technique sensitive to sky projected brightness distributions, constituting a powerful tool for the study of detailed stellar surface structures. Moreover, by combining high spectral and angular resolution we obtain a technique called differential interferometry that is also sensitive to mechanisms that induce chromatic signatures, such as stellar spots and large scale mass motions (e.g. rapid rotation, non-radial pulsations, shear currents produced by hydrodynamical instabilities). We present here a study of the signatures of stellar rotation on differential interferometry observables showing that they are very sensitive to differential rotation and stellar inclination.
\end{abstract}

Roughly speaking optical long baseline interferometry (OLBI) measures the Fourier transform $\tilde{I}(\lambda, y, z)$ of the sky brightness distribution $I(\lambda, y, z) ; y$ and $z$ are angular coordinates describing the sky plane where $y$ is parallel to the projection of the stellar rotation axis onto the sky. For angularly unresolved stars we can perform a Maclaurin expansion of $\tilde{I}(\lambda, y, z)$ retaining only the zero and first order terms. This corresponds to the so called differential interferometry where the zero order term is the stellar spectral flux $F$ and the two first order terms are the photometric barycenter (photocenter $\vec{\epsilon}$ ) of the star.

Fig. 1 shows radial velocity (RV) maps, spectra and photocenters for several models of differentially rotating stars. The results in Fig. 1 were obtained with an interferometry-oriented model including solid or differential rotation, gravity darkening, and local plane parallel atmospheres. This model is further detailed by Domiciano de Souza et al. (2002) who also present an interferometric study of rapidly and uniformly rotating stars.

\section{References}

Domiciano de Souza, A. et al. 2002, A\&A 393, 345

Reiners \& Schmitt 2002, A\&A 384, 155

\footnotetext{
${ }^{1}$ A.D.S. acknowledges CAPES - Brazil (contract BEX 1661/98-1), IAU, and UNSA/UMR6525

- France for financial support.
} 

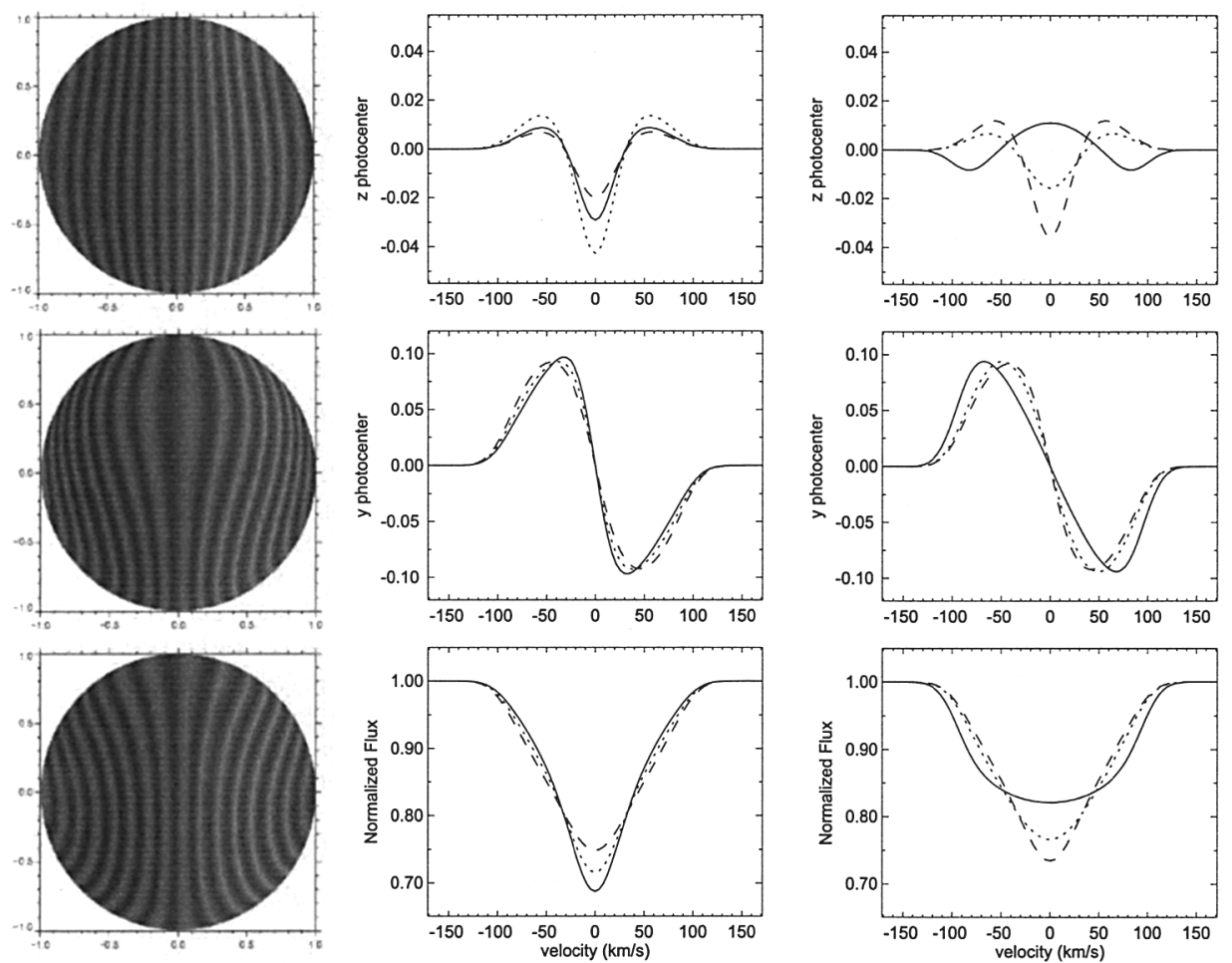

Figure 1. Left: Examples of RV maps for a solar-like differential rotation law, i.e. $\Omega(l)=\frac{v_{\text {eq }}}{R}\left(1-\alpha \sin ^{2} l\right)$, where $R$ is the stellar radius, $\alpha$ is the differential rotation parameter, $l$ is the latitude and $v_{\text {eq }}$ is the linear equatorial rotation velocity. The figure show three different combinations of stellar inclination $i$ and differential rotation rate $\alpha$. $v_{\text {eq }} \sin i$ is fixed to $100 \mathrm{~km} / \mathrm{s}$. Contrarily to rigid rotation regions of constant $\mathrm{RV}$ are not straight lines. Center: Normalized spectrum (bottom) and photocenter components $\epsilon_{y}$ (middle) and $\epsilon_{z}$ (top) for $i$ equal to $20^{\circ}$ (solid), $45^{\circ}$ (dots) and $75^{\circ}$ (dashes). The fixed parameters are $v_{\text {eq }} \sin i=100 \mathrm{~km} / \mathrm{s}$, linear limb darkening $\epsilon=0.6$, local profile given by a gaussian of equivalent width equal to $30 \mathrm{~km} / \mathrm{s}$, and a differential rotation parameter $\alpha=0.6$. Right: Similar to the central column but for $i=60^{\circ}$ with the curves corresponding to $\alpha$ equal to -0.43 (solid), 0.33 (dots) and 0.6 (dashes). The photocenters are given in angular stellar radius units. From this curves it is clear that spectra and photocenters are very sensitive to differential rotation and stellar $i$. Reiners \& Schmitt (2002) studied in details how to detect differential rotation from line profiles. They showed that, although differential rotation can be detected, $\alpha$ and $i$ cannot be disentangled from line profile observations alone. Our first calculations on differential interferometry summarized here indicate that this technique can disentangle $i$ and the differential rotation rate (Domiciano de Souza et al., in preparation). 